\title{
The Function of Aesthetic Education to Medical Students' Professional Aesthetic Literacy
}

\author{
Mi Feng \\ Qiqihar Medical University \\ Qiqihar Heilongjiang Province 161000
}

\author{
Sun Ying \\ Qiqihar Hospital of Traditional Chinese Medicine \\ Qiqihar Heilongjiang Province 161000
}

\author{
Song Yaoxin* Corresponding author \\ Qiqihar Medical University \\ Qiqihar Heilongjiang Province 161000
}

\author{
Chu Zhiwei \\ Heilongjiang Engineering College \\ Harbin Heilongjiang Province 150000
}

\begin{abstract}
Medical aesthetic education is not only an important link in medical education, but also the aesthetic culture that modern college students should possess. It is that the educators, with certain medical aesthetic thoughts and theoretical guidance, promote the formation of the medical students' aesthetics and aesthetic ability and improve, purify and enrich student's thought emotion, set up the correct aesthetic standard, form scientific aesthetical standard, and perception, appreciation and creativity of aesthetics and medical aesthetics, mold perfect personality, so as to gradually form conscious aesthetic value orientation in the medical practice.
\end{abstract}

Keywords-Aesthetic education; Medical students; Professional quality; functions

\section{INTRODUCTION}

Education is to promote the aesthetic development of individuals and the all-round development of human beings through aesthetic education activity, thus achieving the harmonious and perfect development of human and society. Aesthetic education trains students' aesthetic perception and aesthetic experience ability, perfect students' aesthetic taste, allowing them to have the ability to appreciate beauty and create beauty in social life.

In medical colleges, the aesthetic education inspires medical students' feeling of beauty through the image of beauty, arousing their inner emotional resonance, thus inspires them to have correct aesthetic evaluation of objective things, and know the true nature of things, acquiring the ability to understand the true, the good and the beautiful. Medical aesthetic education mainly improves the quality of the students in all aspects through colorful and vivid aesthetic education activities, so that the students' mind is cultivated and their emotion is edified. Develop and enrich students' emotional structure, cultivate the perfect personality, making medical students become all-round developed high-quality medical talent who can correctly understand beauty, appreciate beauty, express beauty and create beauty through medical aesthetic practice.

This article is the research production of Heilongiang Provincial Philosophy and Social Sciences Research and Planning Project "Higher Medical College medical humanities quality training model reform research", approval number: 17EDD180

\section{AESTHETIC EDUCATION AND INDIVIDUAL COMPREHENSIVE DEVELOPMENT}

Strengthen medical aesthetic knowledge education of medical students through the combining of aesthetic theory in aesthetic education with the characteristics of the medical specialty. It plays an important role in building the medical students' correct aesthetic view, establishing perfect emotional education system of medical students, so as to cultivate the healthy personality of medical students and medical practitioners, expand and improve their knowledge structure, and train more complex medical personnel with innovative potential. Continuously improve the professional aesthetic quality of medical students and let it run through the whole process of medical practice. It is of great practical significance to fully reflect the new goals of health and beauty of modern medicine.[1]

\section{A. Promote the harmonious development of medical students' sensibility and rationality}

Medical aesthetic training must begin with sensibility and rationality, and start with beautiful things. Medical workers should first understand the perceptual form of natural beauty, social beauty and artistic beauty, and turn the knowledge into a rational understanding to improve their ability of appreciating beauty, find beauty, and form a love for beautiful things. In this way can we find the elements of beauty in medical activities, and consciously pursue beauty and express beauty. For example, in medical activities, the medical staff is accurate, skilled, and pays attention to the effective communication with the service object, which is the manifestation of the medical personnel's beauty of emotional and rational coordination.

In art appreciation course, for example, students see the image of the works, and combine with the students' awareness of their own to experience and analyze the story behind the works, and at the same time feel the painter's expression of emotional experience, and experience the aesthetic connotation that the author expresses, so as to set up the correct aesthetic values. Thus, aesthetic education can make students enjoy the real life in the process of aesthetic appreciation, and establish the correct aesthetic intention in pluralistic aesthetic values by 
comparing and selecting, so as to establish the correct aesthetic view at heart.

\section{B. Help medical students to achieve the perfect combination of the true, the good and the beautiful}

Medical activities are different from general social practice activities, which are activities to prevent or eliminate diseases and to maintain the health of healthy people. The "real" in medical treatment activity is to follow the law of medicine in the medical service, the "good" in medical treatment activity is the result of medical treatment activity must maintain patients' or other people's physical function. If you leave these things and just seek for the beautiful, there will not be free and pleasant looking in appearance and manner, and will not bring you aesthetic pleasure. Therefore, the medical aesthetic cultivation should eventually make people become the person who seeks truth, goodness and beauty.

The formation of medical aesthetic culture is inseparable from ideology, culture, life, work experience of the aesthetic subject. It is the good emotions and behaviors that we acquire and show from persistent repeated study, experience and accumulation in our long-term social life, medical activities. If we only satisfy with aesthetic education, and ignore the conscious consolidation and applications of the result of the aesthetic education in living and working, we are not able to obtain medical aesthetic accomplishment for ignoring of the active cognitive learning of the good things, or independently appreciate beauty, find beauty, seek beauty, or even create beauty.

\section{IMPROVE THE AEsthetic Cultivation OF MedicAL STUDENTS}

Medical aesthetic accomplishment is that medical students consciously and unconsciously to have long-term aesthetic leaning in working practice, and form a habit. Medical aesthetic culture is the continuation and development of medical aesthetic education. Education is an exogenous motive, while aesthetic cultivation is more about embodiment of conscious aesthetic education behavior, which is manifested as long-term and conscious learning behavior. In the practice of clinical medicine, medical workers should not only attach importance to the aesthetic education in clinical practice, but also pay attention to the medical aesthetic culture.[2]

\section{A. It helps to understand medical aesthetics correctly}

The aesthetic education of medical students refers to the practical process and psychological activity form of understanding, feeling, choosing and judging beauty and ugliness of medical students according to the principle of general aesthetic and standards of medical beauty. It is the basis to use imaginal thinking to create medical beauty in clinical practice.

The ability to find beauty, appreciate beauty and create beauty is cultivated in the long-term social practice, so the medical aesthetic accomplishment emphasize more about that medical students have long-term and continuous learning, discover and pay attention to beauty consciously, so as to improve the sensibility and creativity of medical aesthetics.

\section{B. Promote medical student to feel the beauty scientifically}

The primary task of medical aesthetic training is to train medical staff's ability to fully appreciate the beauty in life. That is to say medical workers respond to the beauty in life through their senses -- aesthetic consciousness, which then becomes the sense of beauty. The medical staff must also learn to feel the medical beauty of medical services on the basis of feeling literature, music, painting, sculpture, etc., such as the beauty of mental quality, sentiment and service behavior of medical service.

\section{Improve the ability to appreciate beauty}

It is the recognition and evaluation of beautiful objects. Most of people's love and appreciation for the beautiful things is spontaneous, simple, elementary appreciation; some even do not conform to the requirements of the aesthetics. Such appreciation level is not enough, so we need to develop our own aesthetic appreciation, form good medical aesthetic appreciation ability. For example, in the plastic surgery, we should not only know what's the most beautiful status of the surgical spot, but also know how to achieve the overall beauty after plastic surgery.

\section{Create beauty in a better way}

The creation of medical beauty has cast medical workers' thoughts, feelings, wills and abilities. Master aesthetic knowledge and grasp the shape and characteristics of medical aesthetic objects to create beauty in medical service. The beauty that medical staff create includes: human's longevity and health, strong and handsome body, sound physiological function, smart mind, and how to better exert various potential of people. All of these are the fields in which the human can create beauty.

\section{Perfect the Connotation of Aesthetic ACCOMPLISHMENT OF MEDICAL STUDENTS}

The main content of medical aesthetic accomplishment refers to that educate and cultivate learners' moral and taste through the element of beauty in medical service activities, and train learners' healthy and elegant aesthetics and ability to feel and create medical beauty. Aesthetic accomplishment of medical practice needs to learn to blend the medical aesthetic theory into clinical medical, nursing, scientific research, medical management, and other activities, so as to apply what one has learned and solve problems in practice, effectively improving the aesthetic quality of the medical staff.[3]

- In medical practice, the aesthetic cultivation is to train medical workers to understand the aesthetic requirements of people in disease, in recovery status or under healthy status; to learn to apply the theory of harmony, balance and integrity of medical aesthetics to guide clinical medical activities; to pay attention to the effects of various elements of beauty on human health and disease; to set the medical aesthetics thought that the aim of medical activities is to maintain the human body beauty, human healthy beauty and vitality beauty, so as to fully understand 
the problems of beauty in the medical field and better maintain the medical beauty of human body.

- The volunteers of aesthetic accomplishment in medical research should pay attention to the beauty of thinking in medical research, clear and organized thinking. The expression of scientific research achievement should be beautiful: accurate language, clear specimen, model and picture. Scientific research methods should be beautiful: rational hypothesis, design, analysis and generalization. The beauty of paper: symmetrical structure, etc.

- The aesthetic cultivation in medical management. Medical management is the "planning - organization implementation - inspection" conducted for medical purposes. Its beauty includes: integrity, the unity of words and deeds, performance of obligations; create harmonious medical and medical care relations; construct beautiful internal and external medical environment; beauty of noble medical ethics and advanced model.

\section{The Core Meaning of Medical Aesthetics EDUCATION}

In terms of aesthetic curriculum, we should attach importance to both the setting of courses of aesthetic education value, and the cultivation of students' aesthetic spirit. It is recommended to increase the cultural background courses and design drawing courses which are related to the specialty, and let students understand aesthetic knowledge of cultural and historical background of the learned specialty, improve culture connotation of the specialty, stimulate students' interest in learning, which in turn can also help students to better learn professional class.

People have gained a better understanding of the world through the transformation of the world, and people have experienced and appreciated beauty through their own practice activities, thus creating beauty and constantly improving their ability to create beauty. Therefore, only to cultivate medical students to master the law of creating beauty, exert the potential to create beauty and consciously apply this to the clinical medical practice. This is the basic task of aesthetics. Therefore, the ability of medical students to create beauty according to the law of beauty in medical practice is a significant and profound task of education of medical schools.

In medical treatment activity, there are a lot of beauties. Some only rely on medical technology to correct, protect and restore people's normal physiology. However, there are a lot of cases only achieving certain physiological targets after treatment. But this is not the only criterion of treatment; we should fully consider the aesthetic effect after treatment. How can we achieve the creation of scientific beauty in medical activities? This depends on the doctor's aesthetics and aesthetic appreciation.[4-5]

\section{The Implementation of Medical Aesthetics EDUCATION}

There are many ways to implement medical aesthetic cultivation, including education on campus and off campus, or self-cultivation in medical work.

School is an important place to implement aesthetic education. Fully use classroom teaching to enable students to learn the basic knowledge of aesthetics, enjoy beauty by appreciating and reviewing beautiful objects, so as to form noble aesthetics and train aesthetic imagination and creativity. In teaching, we can determine the aesthetic education tasks in different stages to meet the individual aesthetic needs and promote the individual aesthetic development. Understand the characteristics of each student's aesthetic development to really make teaching suitable for students' aesthetic needs. By opening related medical aesthetics courses and aesthetic knowledge lecture in theory teaching and experimental teaching, help students set up the correct aesthetic concepts, and train the ability of appreciating beauty and creating beauty by organizing related aesthetic activities.

The aesthetic learning in school is limited, so learners should also insist training their acuity of beauty in life and medical training, actively appreciating beauty, so as to enhance their aesthetic accomplishment, and their ability to create medical beauty. Medical personnel should enhance their own artistic accomplishment and their personal professional ethics, strengthen the aesthetic cultivation of their own language and behavior. In the face of all medical aesthetic objects, actively conduct aesthetic appreciation to gradually form aesthetic feeling. Insist and deepen continuously, so as to improve their aesthetic accomplishment level.

\section{CONCLUSIONS}

The implementation of the aesthetic education of medical students has a preliminary definition of architecture, which fully reflects the multi-level structure of skills, aesthetics and thought in the teaching practice. Aesthetic education of medical students is not a decoration of teaching; instead it is an important means to promote the students' harmonious development of morality, intelligence, physique and aesthetics.

Strengthen medical aesthetic knowledge education of medical students through the combining of aesthetic theory in aesthetic education with the characteristics of the medical specialty. It plays an important role in building the medical students' correct aesthetic view, establishing perfect emotional education system of medical students, so as to cultivate the healthy personality of medical students and medical practitioners, expand and improve their knowledge structure, and train more complex medical personnel with innovative potential. Continuously improve the professional aesthetic quality of medical students and let it run through the whole process of medical practice. It is of great practical significance to fully reflect the new goals of health and beauty of modern medicine. Conduct aesthetic education according to the characteristics of the subject, so as to integrate the pursuit for the beauty, the truth and the good, truly achieve the purpose of both teaching and educating. 


\section{REFERENCES}

[1] M. Cai Yuanpei: Cai Yuanpei's Aesthetic Selections, Peking University Press, 1983. (In Chinese)

[2] M. Cai Yuanpei: The Whole Collection of Cai Yuanpei's Aesthetics, Zhejiang Education Press, 1997. (In Chinese)
[3] M. Wang Defeng. Philosophy of Art, Fudan University Press, 2005. (In Chinese)

[4] M. Shi Xusheng: The Theory of Creative Arts, China Broadcasting and Television Press, 2002. (In Chinese)

[5] M. Wang Feng: Introduction to Medical Aesthetics, Higher Education Press, 2005. (In Chinese) 